\title{
A Survey on Event Detection and Transmission Protocols in an Event Driven Wireless Sensor Network
}

\author{
Krishna Priya.K.C \\ PG Scholar/Dept. of CSE \\ Karunya University
}

\author{
Sebastian Terence \\ Assistant Professor/Dept. of CSE \\ Karunya University
}

\begin{abstract}
The most important goal of Event Driven Wireless Sensor Network (EDWSN) is to detect an event and pass this detected event to the users. Accurate event detection and fast event transmission play vital role in event detection. Many researchers have been proposed various techniques to detect events in an EDWSN. This paper describes a survey on event detection and event transmission protocols in an Event Driven Wireless Sensor Networks. We have classified these protocols into mainly two categories namely Sink Centric Event Detection and Transmission Protocols (SCEDTPs) and Node Centric Event Detection and Transmission Protocols (NCEDTPs). Then, these protocols are further classified into three categories under the constraints reliability, congestion resolution and energy efficiency. The advantages and disadvantages of each protocol also studied and we made the comparison of each protocol with different constraints.
\end{abstract}

\section{Keywords}

EDWSN, reliability, congestion resolution, energy efficiency.

\section{INTRODUCTION}

The main goal of Event Driven Wireless Sensor Network (EDWSN) is event detection and event transmission in a fast and accurate manner. In an EDWSN each of the sensor node is capable of event of detecting different events such as fire detection, enemy detection in battle field, etc., and each node is capable to send the detected events to the sink (Fig.1). The most important benefit of EDWSN is to detect the events where the human beings cannot reach. The main features of EDWSN is number of nodes in a EDWSN can be several orders of magnitude, sensor nodes are densely deployed, sensor nodes are prone to failures, topology of sensor network changes frequently, sensor nodes mainly use broadcast communication paradigm, sensor nodes are limited in power consumption, computational capacities and memory [1]. The heart of the EDWSN is sensor nodes which are deployed in the area and it performs mainly three modules $[1,2]$ namely event detection, event processing and event communication. To perform these three modules efficiently EDWSN should satisfy reliability, congestion control and energy efficiency [3]. The reliability is concerned with how much information is necessary to ensure the occurrence of event in EDWSN. Congestion control deals with reducing traffic in the network and the energy efficiency in EDWSN is necessary to increase its lifetime.

The EDWSN can be node centric or sink centric as shown in Figure 1. In node centric EDWSN the sensor nodes detects, process and finalize the decision based on number of criteria and pass the result to the sink. In case of sink centric EDWSN the sink makes the final decision based on the data received from sink nodes. These two types of sensor networks utilize various protocols for event detection and transmission. And each protocol is characterized by different properties like reliability, congestion resolution and energy efficiency. The application of EDWSN includes military applications, environmental applications, Health application, home application etc [1, 4].

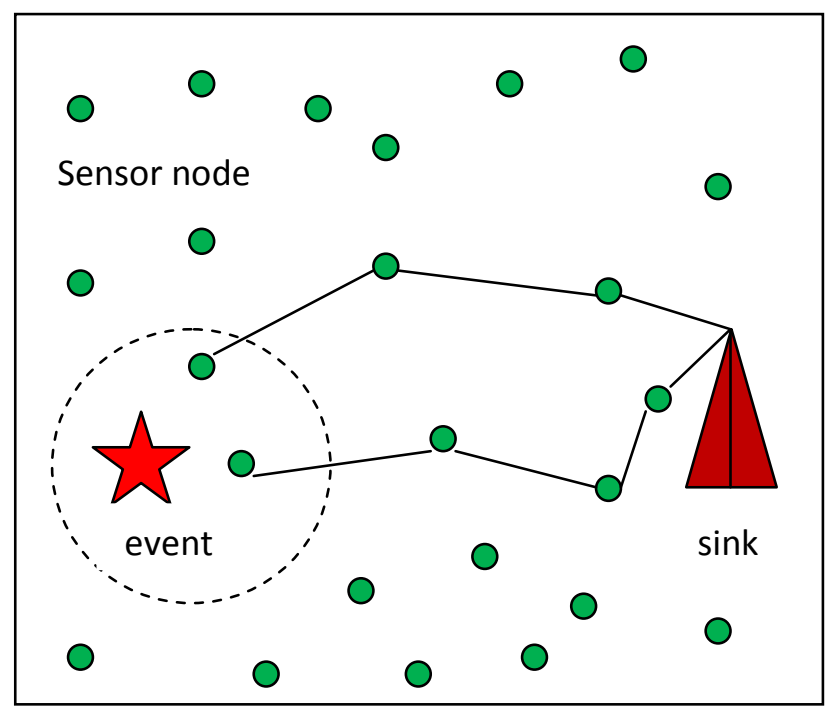

Fig 1. A General EDWSN Scenario

\section{EVENT DETECTION AND TRANSMISSION PROTOCOLS (EDTPS)}

In an EDWSN a number of protocols are used for event detection and transmission called EDTPs. The EDTPs can be mainly classified into Sink Centric EDTPs (SCEDTPs) and Node Centric EDTPs (NCEDTPs), which can be further classified into Reliability Based EDTPs (RBEDTPs), Congestion Resolution Based EDTPs (CRBEDTPs), and Energy Efficiency Based EDTPs (EEBEDTPs). This classification of protocols is as shown in Fig. 2. 


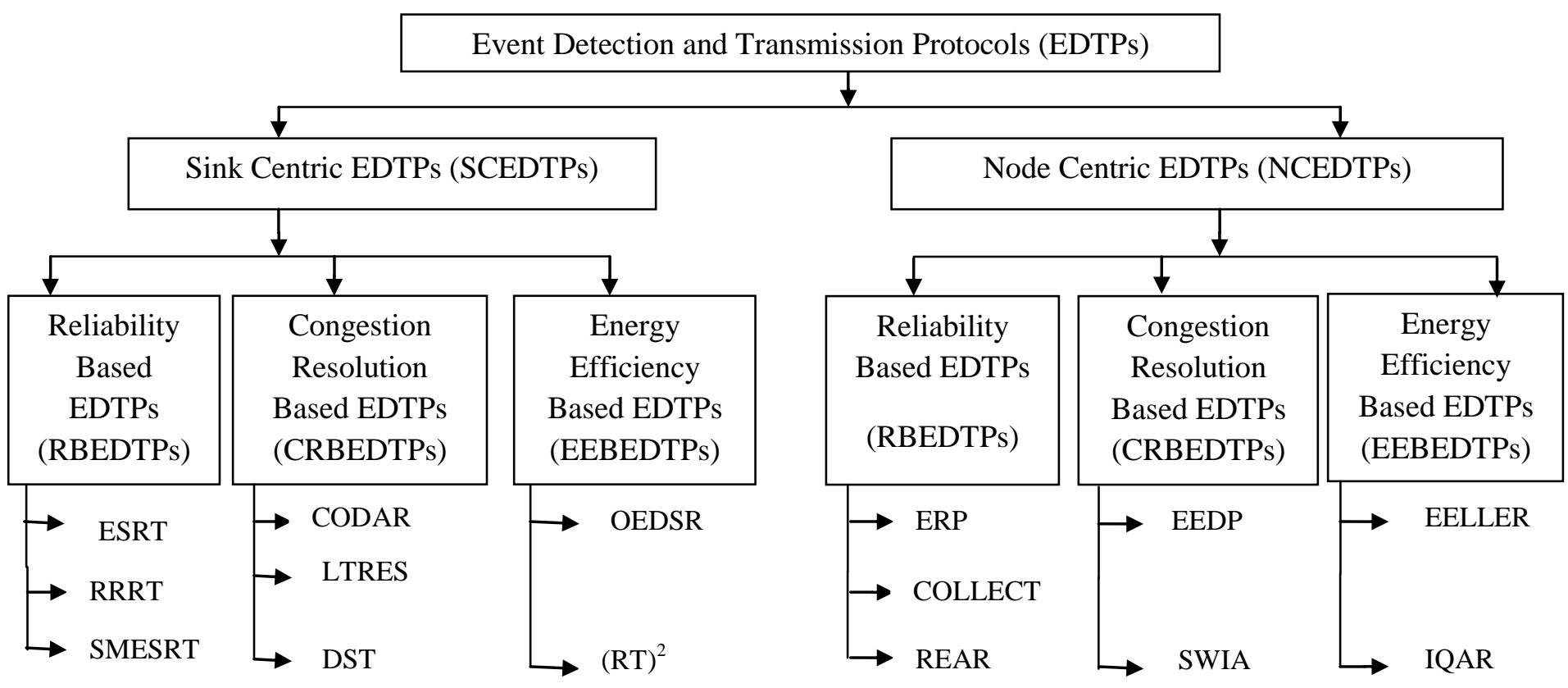

Fig 2.EDTPS in EDWSN

\section{SINK CENTRIC EDTPS (SCEDTPS)}

The sink centric protocols are classified under the constraints of reliability, congestion control and energy efficiency. The sink determines the occurrence of the event based on the sufficient data received from the sensor nodes. The SCEDTPs can be categorized into mainly three classes as such as 1) RBEDTPs, 2) CRBEDTPs and 3) EEBEDTPs.

\subsection{RBEDTPS}

These protocols perform reliable event detection at the sink based on the number of packets received from the sensor nodes using different approaches. The final decision is made in the sink rather than sensor nodes.

\subsubsection{Event-to-Sink Reliable Transport (ESRT) Protocol}

In [5] sink finalize the decision based on collective information from a number of source nodes in the event occurring region rather than individual sensor nodes in the network. ESRT performs the reliable event detection based on reporting frequency $\mathrm{f}$ adjustments under four network states and conditions. Network States/Conditions and the actions performed by ESRT: In each of these conditions the performance of network also vary and these conditions are 1) No Congestion, low reliability (NC, LR)- increase $\mathrm{f}$ multiplicatively, 2) No Congestion, High Reliability (NC, HR)- decrease f conservatively, 3) Congestion, High Reliability (C, HR)- decrease f carefully 4) Congestion, Low Reliability (C, LR)- decrease f exponentially and 5) Optimal Operating Region (OOR)- stay. Advantages of ESRT are Sink only collects information a number of source nodes in the event coverage area and Provide various levels of reliability based on network conditions. The disadvantages of ESRT includes 1) ESRT follows central control method which is not energy efficient and 2) in a sensor field with multiple event occurring at the same time the adjustment of $f$ for all the sensor nodes will not perform better as the events are independent to each other and also the event occurring area may not be same [8].

\subsubsection{Reliable Robust and Real-Time (RRRT) Protocol}

In RRRT protocol [7], author proposes two concepts such as event-to-action delay bound to meet the application specific deadlines and a combined congestion control mechanism which provide the dual purpose of achieving reliability and conserving energy. In event-to-action delay bound it is based on assumptions such as Observed delay-constrained event reliability $\left(\mathrm{DR}_{\mathrm{o}}\right)$ : It is the number of packets received at the sink within a certain delay bound in a decision interval, Desired delay-constrained event reliability $\left(\mathrm{DR}_{\mathrm{d}}\right)$ : It is the minimum number of packets required for reliable event detection within a certain delay bound and Delay-constrained reliability indicator $(\boldsymbol{\alpha})$ : i.e., $\alpha=\mathrm{DR}_{\mathrm{o}} / \mathrm{DR}_{\mathrm{d}}$. If the observed delay constrained event reliability is higher than the reliability bound i.e., $\mathrm{DR}_{\mathrm{o}}>\mathrm{DR}_{\mathrm{d}}$ then the event is reliably detected within a specific delay bound [7]. Advantages of RRRT is 1) Reliable event detection based on event-to-action delay bound and 2) Improved energy conserving based on reporting frequency adjustments. Disadvantage of RRRT is Congestion detection and control mechanisms lead to extra overhead.

\subsubsection{Simultaneous Multiple Event-to-Sink}

\section{Reliable Transport (SMESRT) Protocol}

SMESRT [8] is a protocol designed to accomplish simultaneous multiple reliable event detection with a payload control component that serves the dual purpose of less traffic at the sink and conserving energy. In an WSN the sensor 
nodes who detects the event generates an event ID form a cluster and the event ID can be distributed using in-network data aggregation or using cluster based event identification method or dynamically random event ID assignment method. To balance the energy in the network the $\mathrm{CH}$ role can be rotated among all the sensor nodes [9]. With SMESRT the sensor nodes detects the same event sends the event packets to $\mathrm{CH}$ at a predefined reporting frequency for that particular period or duration. The $\mathrm{CH}$ informs the sink about the network condition. The SMESRT performs the payload control at the CHs. Advantages of SMESRT includes 1) payload control at the $\mathrm{CHs}$ provide less traffic and 2) SMESRT can assign different reporting frequency for different events. Disadvantage of SMESRT is assignment of different reporting frequency leads to extra overhead.

\subsection{CRBEDTPS}

These protocols perform event detection and transmission under various congestion or traffic conditions. The protocols coming under this category tries to reduce the packet loss using different mechanisms. The main goal of these protocols is to ensure the congestion resolution under various network states and tries to reduce the traffic using various methods. Some examples are given as follows:

\subsubsection{Congestion and Delay Aware Routing (CODAR) Protocol}

The main objective of CODAR is to improve reliability and the timeliness of the data transmitted by critical nodes through congestion avoidance and mitigation [10,11]. The CODAR protocols classify the sensor nodes into two categories the critical nodes, that is the nodes closer to the event and the regular nodes, i.e., when there is no event sensing occurs the critical nodes become regular nodes. The CODAR mainly performs two mechanisms congestion avoidance and end-toend delivery delay management. Congestion avoidance: Each node in event occurring region broadcasts its location and Relative Success Rate (RSR) value using control packets during a fixed interval [10]. The RSR helps to mitigate congestion by choosing lightly congested nodes. End-to-end delivery data management: In the CODAR, in end-to-end delivery management each critical node transmits their critical data packet with the deadline in its header to the sink. All the intermediate node check this header field before forwarding the packet and if the intermediate node has end-to-end delay that cannot meet the packet's deadline then the intermediate node drops the futile packets. Advantages of CODAR are 1) CODAR delivers high amount critical data within specified delays and 2) CODAR has a potential to reduce congestion by avoiding congested nodes during route selection process and also by dropping futile packets [10]. Disadvantages of CODAR includes 1) not suitable for large number of critical nodes and 2) less energy efficient.

\subsubsection{Loss Tolerant Reliable Event Sensing}

\section{(LTRES) Protocol}

The LTRES performs the congestion control based on the node-end source rate adaptation mechanism: Node-end Distributed Source Rate Adaptation: The sink calculates the Event-sensing Fidelity level $\left(\mathbf{E S F}_{\mathbf{E}}\right)$ and sends the event sensing reliability measure to the Enodes. Based on this Enodes update their source rate in order to ensure the congestion control. It involves mainly three stages. Stage one: In this stage before any congestion detection each node in Enodes perform multiplicative increase (MI) operation on source rate adaptation to approach $\operatorname{ESF}_{\mathrm{E}}=1[5,6]$. Stage two: If any local congestion is detected by the sink due to MI operation before the end of the first stage Enodes enter into stage two. And the congested Enodes perform available bandwidth detection and provide upstream congestion avoidance [6]. Stage three: In EDWSN if there is no active Enodes stop the node-end source rate adaptation and the Enodes provide best effort service with no congestion. Advantages of LTRES includes 1) Performs network traffic control based on distributed source rate adaptation and 2) LTRES performs fast and reliable event sensing compared with LSR protocols. The main disadvantages of LTRES Less energy efficient due to source rate adaptation mechanism.

\subsubsection{Delay Sensitive Transport (DST) Protocol}

The main objective of the DST protocol is to timely and reliably transport event features from the sensor field to the sink with minimum energy consumption and no congestion [12]. The DST employs a Time Critical Event First (TCEF) scheduling mechanism at the sink to meet the application specific delay bounds. The DST mainly involves two mechanisms 1) Congestion control and detection mechanism. In this for any sensor node whose buffer overflows due to excessive incoming packets is said to be congested and it informs the sink congestion condition by a Congestion Notification $(\mathrm{CN})$ bit the event packet header $[12], 2)$ Real-time event transport mechanism: DST performs real-time event detection under event-to-sink delay bound. The main components of event -to-sink delay bound are event transport delay and event processing delay [7, 12]. According to DSRT, for reliable and timely event detection the event-to-sink delay bound should be greater than or equal to the sum of both event transport delay and event processing delay. To achieve this criterion the sink node performs TCEF scheduling policy at the sink. The TCEF policy applies the general principles of earliest deadline first service and on each sensor node, which is an optimal scheduling policy when considering the real-time deadlines of the system [13]. Advantage of DST is DST performs congestion detection and control. The disadvantage of DST is the reporting frequency rate adjustment mechanism leads to overhead.

\subsection{EEBEDTPS}

These protocols perform event detection and transmission with low energy consumption. Some examples for these types of protocols are given as follows: 


\subsubsection{Optimized Energy-Delay Sub-network Routing (OEDSR) Protocol}

The OEDSR, the nodes are either in idle or sleep mode, but once an event is detected, the nodes near the event become active and start forming sub-networks and this formation of inactive network into a sub-network saves energy because only a portion of the network is active in response to an event [14]. The active nodes itself form cluster and choose Cluster Heads $(\mathrm{CHs})$. The packets from $\mathrm{CHs}$ are sent to the sink through relay nodes. The active nodes in the network sends the HELLO message to neighbor nodes which consists of fields such as the active node ID, energy availability of the node, and the sensed attribute. Based on this information the nodes in the network forms cluster so that efficient data aggregation is possible which helps to reduce the energy consumption [15]. Initially a Temporary Head (TH) is selected based on maximum energy and the TH choose the $\mathrm{CHs}$ based on $\mathrm{CH}$ selection factor, which is the product of energy available and the value of the sensed attribute from the event. After the $\mathrm{CHs}$ has been selected the $\mathrm{TH}$ sends a $\mathrm{CH}$ SELECT packet to all the active nodes in the network, subsequently $\mathrm{TH}$ becomes a regular node. When each node receives a beacon from the $\mathrm{CHs}$ the nodes measures the Received Signal Strength Indicator (RSSI) for that particular beacon and also depends on strength of each signal, the node joining into a particular cluster. Advantages of OEDSR include 1) cluster formation leads to less energy consumption and 2) Sub-networks saves energy because only a portion of the network is active. The main disadvantage of OEDSR is the number of packets is transmitted between the sensor nodes, THs and CHs leads to delay.

\subsubsection{Real-Time and Reliable Transport $(R T)^{2}$ \\ Protocol}

The main objective of the (RT) $)^{2}$ protocol is to reliably and collaboratively transport event features from the sensor region to the sink with minimum energy dissipation and to timely react to sensor information with the right action [16]. The $(\mathrm{RT})^{2}$ protocol concurrently provides real-time communication and addresses various reliability requirements which will leads to less energy consumption. There are two nodes are used by this protocol, the sensor nodes and actor nodes. When the sensor nodes detect an event they send the sensed information to the actors in the network. The actor nodes then communicate with each other to make a decision cooperatively and then send the event packet to the sink by actor nodes. The (RT) ${ }^{2}$ protocol uses the new idea of event-toaction delay bound which is the sum of the event transport delay, event processing delay, and action delay [7, 16]. The $(\mathrm{RT})^{2}$ protocol operation is based on two states namely StartUp state: In this state the sender transport a probe packet toward the receiver to capture available transmission rates and Steady State: It consists of four sub states namely increase, decrease, hold and probe. During the increase state the sender increases the transmission rate according to the feedback from the sink. In the decrease state the sender reduces its transmission rate according to the feedback coming from the sink. The hold state is achieved when the required transmission rate is achieved. In probe state the sender sends a probe packet to the sink in order to monitor the available transmission rate in the network. Advantages of $(\mathrm{RT})^{2}$ includes 1) the $(\mathrm{RT})^{2}$ protocol adjusts its configurations in order to adapt to the heterogeneous characteristics of wireless sensor networks and 2) provides timely reaction. The Disadvantage of $(\mathrm{RT})^{2}$ is the configuration adjustment nature of $(\mathrm{RT})^{2}$ leads to extra delay.

\section{NODE CENTRIC EDTPS (NCEDTPS)}

By using these protocols the sensor nodes makes the final decision from the data received to it and send their result to the sink. The NCEDPs can classified into three categories as in SCEDPs and each of these protocols performs event detection and transmission under various constraints such as reliability, congestion control, and energy efficiency such as 1) RBEDTPs, 2) CRBEDTPs, and 3) EEBEDTPs

\subsection{RBEDTPS}

These types of protocols perform event detection among the sensor nodes itself. The decision may be made by one or more nodes. After the event detection the sensor nodes performs transmission of the detected event using different forwarding techniques. A brief overview of these protocols is discussed as follows.

\subsubsection{Event Reliability Protocol (ERP)}

The main objective of the ERP is to perform reliable transfer of packets containing information about an event to the sink while minimizing similar redundant packets [17]. The ERP protocol uses an implicit acknowledgement (iACK) mechanism to achieve the reliability. The ERP works based on a mechanism called Region-based selective retransmission mechanism: When the sensor nodes senses the event it will send the detected event to the next hop node and then this next hop node put the event packet in its buffer and the packet at the head of its buffer is transmit to the next hop. When the node hears that the next hop node transmitting the packet it has sent, it is an implicit acknowledgement that the packet is forwarded successfully [17]. And the node then removes the packet from the queue end and the next packet in the queue is processed. The region-based selective retransmission is based on the source ID, source location and event's time at a source of a particular packet to be retransmitted. By observing the packets in the queue of a particular node the distance between the nodes are calculated and check whether they are in they are in-range of one another. If there is another packet from the same event region send that packet to the sink else send the first packet again until a packet from the same event region is reached to the node's queue. Advantages of ERP are 1) reliable event transmission and 2) minimize redundant transmission of data packets. The disadvantage of ERP is less energy efficient.

\subsubsection{COLLaborative Event deteCtion and Tracking (COLLECT) protocol}

The COLLECT consists of three procedures vicinity triangulation, event determination, and border sensor 
selection. The vicinity triangulation procedure enables the same kind of sensor to construct the respective attribute region, named logical triangle to accurately identify the event region [18]. In event determination procedure a sensor node locally determines the existence of the event according to its sensor data and received messages from the different kinds of sensors within its logical triangles and the border selection procedure aims to select the border sensors to stand for the event boundary [18]. The COLLECT understand the role of sensors by identifying the status of the sensor which includes ordinary, alert, and urgent. A sensor is called ordinary if it does not sense any event attribute. An alert sensor is one if it perceives any kind of event attribute. A sensor is called urgent if the event occur in its coverage area or sensing area. When an ordinary node detects an event it becomes an alert node and send an ATR packet to its neighbours which consists of the ID of the sensor node, its location, the attribute it detected, and the and the timestamp when it detects the event attribute. Based on this information the sensor nodes participate in the vicinity triangulation. Advantages of COLLECT are 1) collect is a fully distributed scheme and 2) COLLECT enables sensor

Table 1. Comparison Of EDWNS Protocols

\begin{tabular}{|c|c|c|c|c|c|c|c|}
\hline \multicolumn{8}{|c|}{ EDTPs } \\
\hline PROTOCOLS & $\begin{array}{c}\text { SINK } \\
\text { CENTRIC }\end{array}$ & $\begin{array}{c}\text { NODE } \\
\text { CENTRIC }\end{array}$ & $\begin{array}{c}\text { RELIABILITY } \\
\text { BASED }\end{array}$ & $\begin{array}{l}\text { CONGESTION } \\
\text { RESOLUTION } \\
\text { BASED }\end{array}$ & $\begin{array}{c}\text { ENERGY } \\
\text { EFFICIENT } \\
\text { BASED }\end{array}$ & $\begin{array}{c}\text { LOSS } \\
\text { RELIABILITY }\end{array}$ & $\begin{array}{c}\text { LOSS } \\
\text { RECOVERY }\end{array}$ \\
\hline ESRT & YES & NO & $\checkmark$ & & & & END TO END \\
\hline RRRT & YES & NO & $\checkmark$ & & & SACK & END TO END \\
\hline SMESRT & YES & NO & $\checkmark$ & & & ACK/NACK & END TO END \\
\hline CODAR & YES & $\mathrm{NO}$ & & $\checkmark$ & & $\sim$ & END TO END \\
\hline LTRES & YES & NO & & $\checkmark$ & & ACK/NACK & END TO END \\
\hline DST & YES & NO & & $\checkmark$ & & & END TO END \\
\hline OEDSR & YES & $\mathrm{NO}$ & & & $\checkmark$ & ACK/NACK & END TO END \\
\hline$(\mathrm{RT})^{2}$ & YES & $\mathrm{NO}$ & & & $\checkmark$ & SACK & END TO END \\
\hline ERP & $\mathrm{NO}$ & YES & $\checkmark$ & & & iACK & HOP BY HOP \\
\hline COLLECT & $\mathrm{NO}$ & YES & $\checkmark$ & & & $\sim$ & HOP BY HOP \\
\hline REAR & $\mathrm{NO}$ & YES & $\checkmark$ & & & $\mathrm{ACK}$ & \\
\hline EEDP & NO & YES & & $\checkmark$ & & r & HOP BY HOP \\
\hline SWIA & YES & $\mathrm{NO}$ & & $\checkmark$ & & $\bar{z}$ & HOP BY HOP \\
\hline EELLER & $\mathrm{NO}$ & YES & & & $\checkmark$ & $\sim$ & HOP BY HOP \\
\hline IQAR & NO & YES & & & $\checkmark$ & 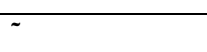 & HOP BY HOP \\
\hline
\end{tabular}


to promptly detect and track the event. The main disadvantage with COLLECT is it cost-effective because of no need of sensor redeployment.

\subsubsection{Reliable Energy Aware Routing (REAR) Protocol}

In [19] it has been proposed that the REAR protocol allows each sensor node to confirm sensor node to confirm success of data transmission to other sensor nodes by supporting the DATA-ACK oriented packet transmission. The sink node sends the ACK packet to the sensor nodes which indicates the successful receiving of event packets. If the sensor node receives the ACK within the ACK timeout, it transmit new sensing event packet. If ACK does not receives within the desired time it considers it as transmission error and it assumes that the event packet which already has been send is lost. If data transmission to next hope fails the node sends an error message packet to the source node. Then the source node receiving the error message resends it with a second path and thus the reliability of data transmission is guaranteed [19]. The REAR involves the components like sender, receiver, receiver event handler, queue manager, routing manager, REAR checker and delay estimator. Advantage of REAR are provides routing. The Disadvantage with REAR is the use of queues and estimators leads to extra overhead.

\subsection{CRBEDTPS}

These are the event detection protocols which perform event detection and transmission with less congestion. The decision about an incident is made in the sensor nodes itself. These protocols work based on various mechanisms in order to reduce the traffic. The protocols coming under this category includes:

\subsubsection{Efficient Event Detection Protocol (EEDP)}

The main objective of EEDP is to fast transmission of the event packet from the decision made node to the sink node with reduced traffic. With this protocol the sensor nodes nearer to the event occurring region senses the event and makes their own decision based on Simple Decision Rule (SDR) that is whether the event has happened or not and makes further accurate decision based on Composite Decision Rule (CDR) [20]. The event reliability is achieved by using a method called dynamic multi-copy scheme. EEDP is mainly based on two procedures Primary Detection Procedure (PDP) and Emergency Routing Procedure (ERP). In PDP the accurate event decision is made based on SDR and CDR and in ERP the event packet is forwarded to the sink by using greedy approach and for reliability simple dynamic multicopy scheme has been used. Advantages of EEDP include 1) accurate event detection and 2) no significant amount of data is send to the sink. The disadvantage with EEDP is less reliability due to only one decision node sends the event information to the sink.

\subsubsection{Stop-and Wait-Implicit Acknowledgement (SWIA) Protocol}

The SWIA protocol does not allows the sensor node to send the next packet before it received an ACK packet for the previous packet. The SWIA protocol makes the use of implicit acknowledgement [21]. The iACK mechanism exploits the broadcast nature of wireless sensor network. In iACK mechanism vthe sensor node after transmitting the packet listens to the channel transmission of packets and thus reduce network traffic. Advantages of SWIA include 1) no additional packet overhead and 2) reduced traffic by avoiding unnecessary event packet transmission. Disadvantage of SWIA is the use of iACK mechanism provides some delay in the network performance.

\subsection{EEBEDTPS}

In a WSN, the sensor nodes make the decision about an event with less energy. In order to support this number of protocols is used which mainly involves:

\subsubsection{Energy Efficient-Low Latency Express Routing Protocol (EELLER)}

The EELLER protocol is a hierarchical routing protocol based on clustering to minimize the number of hops required for data reporting as well as achieving energy efficiency [22]. During the event detection the detected data is forwarded hopby-hop through cluster heads. The EELLER uses hierarchical routing to perform energy-efficient routing in an event driven wireless sensor networks. In hierarchical routing procedure the high energy nodes are used to send the information and the low energy nodes is used to sense the event. The EELLER mainly consists of two phases constructing expressways and cluster formation and data communication. During the first phase the first hope and the second hope is selected based on the link factor, where link factor is the ratio of the energy of the node to the distance to the sink. During the second phase of EELLER, it provides a better data transmission after data aggregation and removing redundancy by the cluster heads. Advantages of EELLER include 1) energy efficient and 2) data aggregation provides more event accuracy. Disadvantage of EELLER is less reliability.

\subsubsection{Information Quality Aware Routing (IQAR) Protocol}

Unlike other aggregation-based schemes IQAR consider the information content of data during data aggregation and forwarding [23]. The IQAR protocol has been adopted tree based approach and its objective is to detect event in a sensor network. The information quality is concerned with the accuracy of the event information. In this each sensor nodes independently detects and collects data about an event and makes a per-sample binary decision i.e., whether the event has happened or not. If there is an event the result of per-sample binary decision will be 1 else 0 . After this a global For pages other than the first page, start at the top of the page, and continue in double-column format. The two columns on the last page should be as close to equal length as possible.

\section{CONCLUSION}

In this paper we studied different EDTPs in EDWSN and we classified these protocols into mainly two types namely SCEDTPs and NCEDTPs. In SCEDTPs the event detection and the decision about a particular event is performed by the sink while in NCEDTPs the event decision is made among the sensor nodes. In each of these classifications the protocols can be again classified into RBEDTPs, CRBEDTPs, and EEBEDTPs. The RBEDTPs perform the event detection 
under reliability and the CRBEDTPs detect an event by considering the traffic conditions of the network. The EEBEDPs perform event detection by considering the energy efficiency. Each of these protocols performs the event detection and transmission based on various approaches.

\section{REFERENCES}

[1] Akyildiz, W.Su, Y. Sankarasubramanian, and E. Cayirci,“A survey on sensor networks”, IEEE Commun. Mag., vol.40, no.8, pp. 102-114, August, 2002.

[2] Muhammad AdeelMahmood, WintsonSeah, "Reliability in Wireless Sensor Networks: Survey and Challenges Ahead", ELSEVIER JOURNEL, February 8, 2012

[3] S.Kamal, P.varalakshmi, "Energy Efficient and Congestion Avoidance Event Tracking in Wireless Sensor Networks", ICSCCN, pp. 167-171, 22 July 2011.

[4] Neha Singh, Prof.RajeswarLal Dua, Vinita Mathur, "Wireless Sensor Networks: Architecture, Protocols, Simulator Tool", IJARCSSE, vol.2, pp. 229-233, May.2012.

[5] O. B. Akan and I. F. Akyildiz, "ESRT: Event-to-sink reliable transport in wireless sensor networks", IEEE/ACM Transactions on Networking, vol. 13, no. 05, pp. 1003-1016, 2005

[6] Y. Xue, B. Ramamurthy and Y. Wang, "LTRES: A losstolerant re-liable event sensing protocol for wireless sensor networks", Computer Communications, vol. 32, no. 15 , pp. $1666-1676,2009$.

[7] Deepali Virmani, Stabirjain, "Reliable Robust and RealTime Communication Protocol For Data Delivery in Wireless Sensor Networks", IJITKM , vol.4, pp. 595601, Dec.2011.

[8] HafizurRahman, DebajyotiKarmaker, Mohammad Saiedur, Rahman, Nahar Sultana, "SMESRT: A Protocol For Multiple Event-To-Sink Reliability In WSN", IJET, vol.1, no.1, pp. 9-14, October.2011.

[9] NizarBouabdallah, Mario E., "Continuous Monitoring Using Event-Driven Reporting for Cluster-Based WSN", IEEE Transaction on Vehicular Technology, vol.58, no.7, pp. 3460-3479, September,2009.

[10] Mohammad M, IqbalGonda, Jordar K, "CODAR: Congestion and Delay Aware Routing to Detect Time Critical Events in WSNs", IEEE, 2011.

[11] M.M Bhuiyan, Gondal, J. Kamrazzuaman, "Congestion Avoidance and Mitigation in WSN", IEEE, May,2010.
[12] V. C. Gungor and O. B. Akan, "DST: delay sensitive transport in wirelesssensor networks", in Proceedings of the 7th International Symposium on Computer Networks (ISCN), Istanbul, Turkey, pp. 116-122, 16-18 June2006.

[13] M. Schwartz, "Mobile Wireless Communication", IEEE,vol.6, pp. 356-368, December,2011.

[14] Sibila R, Sarangapani J, Vittal R, "OEDSR: Optimized Energy-Delay Sub-network Routing in WSN", IEEE, pp. 330-335, 2011.

[15] Luis J.G.V, Ana L.S.O, Alicia T.C, Claudia J.B.A, "Routing Protocols in Wireless Sensor Network", SENSORS JOURNEL, pp. 8399-8421, 2009.

[16] Vehbi C.G, Ozgur B.A, Ian F.Akyildiz, "A Real-Time and Reliable Transport (RT) ${ }^{2}$ Protocol for WSN and Actor Networks", IEEE/ACM Transactions On Networking, vol.16, no.2, pp. 359-370, April 2008.

[17] M. A. Mahmood and W. K. G. Seah, "Event Reliability in Wireless Sen-sorNetworks",in Proceedings of the seventh International Conference on Intelligent Sensors, Sensor Networks and Information Processing (ISS-NIP), Adelaide, Australia, pp. 01-06, 06-09 December 2011.

[18] Kuei-Ping S, Sheng-Shih W, Pao-Hwa Y, Chau-Chieh, "CollECT: Collaborative Event detection and Tracking in Wireless Heterogenous Sensor Networks",IEEE (ISCC '06), 2006.

[19] Kee-Young S, Junkeun S, JinWon K, Misun Yu, PyeongSooMah, "REAR: Reliable Energy Aware Routing Protocol for WSN",ICACT, Feb.2007.

[20] Lulu L, Deyun G, Hongke Z, Oliver W.W.Y, "Efficient Event Detection Protocol in Event-Driven WSN," IEEE Sensor Journal, vol.12, no.6, pp. 2328-2337, June.2012.

[21] M. Mar oti, "Directed od-routing framework for wireless sensor net-works", in Proceedings of the 5th ACM/IFIP/USENIX International Conference on Middleware (Middleware), Toronto, Canada, pp. 99114,18-22 October 2004.

[22] Ali M, Fazard T,Fazard T, Mohammad H.Y.M, Mohsen T.H, "EELLER: Energy-Low Latency Express Routing for WSNs", IEEE,vol.3, pp.334-339, 2010.

[23] Hwee-Xian T, Mun-Choon C, Wendong Xiao, PengYong Kong, Chen-Khong T, "Information Quality Aware Routing in Event-Driven Sensor Networks", IEEE INFOCOM, 2010. 